\title{
Study of emergency laboratory parameters in acute oraganophosphorus poisoning in a rural population- retrospective study
}

\author{
Chandana G1, Kavita P Rasalkar ${ }^{2, *}$, Gajjehalli Saikiran Reddy ${ }^{3}$, Vijitha Thinakaran ${ }^{4}$
}

\begin{abstract}
${ }^{\mathbf{1}}$ Assistant Professor, ${ }^{\mathbf{2}}$ Associate Professor, Dept. of Biochemistry, PES Institute of Medical Sciences and Research, Kuppam, Andhra Pradesh, ${ }^{3,4}$ Junior Resident, ${ }^{3}$ Dept. of Emergency Medicine, ${ }^{4}$ Dept. of Pathology, PES Institute of Medical Sciences and Research, Kuppam, Andhra Pradesh, India
\end{abstract}

*Corresponding Author:

Email: drrasalkar27@yahoo.co.in

\begin{abstract}
Introduction: Organophosphorus poisoning (OPP) is one of the common medical emergencies among rural population in India due to suicide attempts. The purpose of this study was to investigate the relationship between the initial emergency laboratory parameters (electrolytes, liver function test, random blood glucose (RBS), renal function test, Hb, RBC and WBC) and severity of organophosphate poisoning based on Bardin's Classification and type of OP compound consumed in patients admitted to emergency department with OPP.

Materials and Methods: A retrospective observational study was done in OPP patients admitted to emergency medicine department. Based on Bardin's classification all OPP cases were classified into 3 Grades of severity of poisoning. ${ }^{1-3}$ Hematological and biochemical parameters were compared based on this severity of poisoning.

Results: With random sampling of 45 patients, 19 male and 26 female patients aged between 25-35 years, admitted with OPP were included in the study. The severity of poisoning was worst in females. The pseudocholinesterase, potassium and creatinine levels were significantly low in patients of grade 3 than grade 1 and grade 2 ( $p<0.05,0.05$ and 0.02 respectively) poisoning. Liver enzymes (AST, ALT and ALP) were elevated in Grade 3 poisoning patients much higher than Grade 1 and Grade 2 (p value $0.004,0.002$ and 0.06 respectively). Hyperglycemia was significantly observed in Grade 3 poisoning patients at the time of admission. ( $\mathrm{p}$ value 0.03 ). There was significant hypokalemia (0.02), low RBC counts $(0.02)$ and pseudocholinesterase levels ( $\mathrm{p}$ value 0.01 ) in patients who had consumed chlorpyriphos and phorate.

Conclusion: Emergency laboratory parameters in acute oraganophosphorus poisoning reflect the need for basic diagnostic facility in rural area. Laboratory parameters can also serve as possible predictive markers for severe outcomes and prognosis in OP poisoning. The knowledge of type of OP compound consumed can predict the severity of OP intoxication and need for emergency intervention.
\end{abstract}

Keywords: Oragano phosphorous poisoning, Biochemical parameters.

\section{Introduction}

Organophosphorus (OP) compounds are employed for pest control worldwide. In developing countries like India due to rampant use of pesticides, short term organophosphorus poisoning (OPP) is one of the common medical emergencies and is not only a leading cause of mortality but also increases the morbidity in recovered patients. They represent an important source of suicidal poisoning among rural population in India in present days because of its easy accessibility and availability.

OP compounds are lipophilic in nature therefore they are quickly absorbed through the respiratory system, skin and gastrointestinal tract mucosa. Phosphate radicals of OP compounds bind to cholinesterase active sites by covalent binding. This binding makes them inert resulting in overstimulation of muscarinic and nicotinic receptors at the synaptic junctions within the central and peripheral nervous system. ${ }^{1,8}$ This produces an array of symptoms like miosis, bradycardia, increased gastrointestinal motility, emesis, sweating, tachypnoea, salivation, lacrimation, altered sensorium, fasciculation, bronchospasm, blurred vision, urination and defecation. If unattended it leads to complications that include acidosis, respiratory paralysis, acute renal failure, seizures, arrhythmia, aspiration etc and finally death due to combination of these complications. . $^{1,2,8}$

In addition to increased cholinergic manifestation, biochemical alterations like hypokalemia, hyperglycemia, and acute renal failure, transient elevation of liver enzymes, abnormal arterial blood gas analysis, and dearranged calcium and phosphorus parameters occur in OP poisoning.

OPP induces oxidative stress leading to pancreatic damage thereby exhibiting glucose intolerance. Apart from oxidative stress, glucocorticoid receptor activation and adrenergic receptor activation causes hyperglycemia via induction of liver gluconeogenesis. ${ }^{3}$

OPP causes adverse effects on tissues thereby causing acute organ failure. Transient renal injury is caused by direct action of the organophosphate, causing tubular cell necrosis or secondary mechanism that follows the cholinergic crisis, leading to hypovolemic shock and rhabdomyolysis. ${ }^{4}$

Bardin's classification for OPP is convenient to be used for early assessment of OPP and grading may 
facilitate to recognize seriously poisoned patients for early emergency care. ${ }^{5}$

Moreover, laboratory confirmation of the diagnosis may be necessary in order to provide optimal patient care. Emergency laboratory tests may give information about first acute organ damage and the degree of poisoning in patients with acute OPP.

The purpose of this study was to investigate the relationships between the initial emergency laboratory parameters (electrolytes, liver function test, random blood glucose (RBS), renal function test, $\mathrm{Hb}, \mathrm{RBC}$ and WBC) and severity of organophosphate poisoning based on Bardins Classification and type of OP compound consumed in patients admitted to emergency medicine department with OPP.

\section{Materials and Methods}

A retrospective observational study was done in collaboration with emergency medicine department at PES Institute of Medical Sciences and research, Kuppam, Andhra Pradesh. Approval from Institutional Research Committee (IRC) \& Institutional Human Ethics Committee (IHEC) was obtained. Patients with organophosphate poisoning admitted to emergency care room from Jan 2014 to Dec 2014 were included in the study.

All poisoning cases (unique hospital identification (UHID) numbers) which were recorded in the emergency medicine department were noted. All OPP cases of both genders aged $>18$ yrs were included in the study and the data related to hematological and biochemical parameters were collected from the Medical records department. Patients other than OPP and known diabetic mellitus were excluded from the study.

Based on Bardin's Classification all OPP cases were classified into 3 Grades of severity of poisoning $(0,1,2$, and 3). Hematological and biochemical parameters were compared based on this severity of poisoning.

Bardin's Classification: The degree of organophosphate intoxication. ${ }^{6}$

Grade 0: No clinical manifestations

Grade 1: Hypersecretion, fasciculation, consciousness
Grade 2: Grade $1+$ hypotension, unconsciousness

Grade 3: Grade 2 + stupor, abnormal chest $\mathrm{x}$-ray, $\mathrm{pO} 2<$ $10 \mathrm{mmHg}$

Serum sample was used to analyze biochemical parameters like LFT (Total Bilirubin, Total protein, albumin, Globulin, AST, ALT and ALP) and RFT (Urea, Creatinine), pseudocholinesterse, Serum Electrolytes (Sodium, Potassium and Chloride). Sodium fluoride and sodium EDTA sample was used for RBS and potassium EDTA sample was used for hematological parameters like $\mathrm{Hb}, \mathrm{WBC}$, and RBC.

All biochemical investigations were analyzed in Vitros 250 auto analyzer and hematological parameters were analyzed in ABX Micro 60 and Sixmax 1000i autoanalyzer.

Statistical Methods: Results are presented as Mean \pm SD. Analysis of variance (ANOVA) was used for the comparison of the three groups. Pearson's correlation coefficient was used for the correlation. Statistical analysis was performed using the statistical package of social sciences (SPSS-15). P values of $<0.05$ were considered as statistically significant.

\section{Results}

With random allocation 105 poisoning cases were selected for inclusion in the study. Due to unavailability of information or cocktail poisoning few cases were excluded. The exact type of the OP compound consumed was known only in 45 patients and they were included in the study. Among 45 patients, 19 were males and 26 were female patients and all were aged between 25-35 years. The severity of poisoning was worse in females than in males; with 13 out of 26 female patients were admitted in critical conditions. 18 patients were placed in Grade 1, 7 patients in Grade 2 and 20 patients in grade 3 . There were no patients in Grade 0. All patients consumed the OP poison orally and mode of poisoning was suicidal. (Table 1).

The common reasons for suicide in these patients were mainly due to financial issues and domestic violence. All patients belonged to low socioeconomic status and were found to be from rural background.

Table 1: Demographic parameters

\begin{tabular}{|l|c|c|c|c|}
\hline \multicolumn{1}{|c|}{ Characteristic } & Grade 1 & Grade 2 & Grade 3 & Total \\
\hline Age & $26.83 \pm 12.62$ & $35.54 \pm 13.42$ & $30.53 \pm 10.11$ & \\
\hline Male & 10 & 2 & 7 & 19 \\
\hline Female & 8 & 5 & 13 & 26 \\
\hline Mode of poisoning & Oral & Oral & Oral & \\
& Suicidal & Suicidal & Suicidal & \\
\hline Total & 18 & 7 & 20 & 45 \\
\hline
\end{tabular}

The average serum pseudocholinesterase levels in patients of grade 3 were revealed to be $(200 \pm 0.00)$ IU/L, which was significantly lower than grade 2 and grade 1 (p <0.05).Likewise potassium also was significantly low in grade 3 severity of poisoning than in Grade 1 and 2 ( $\mathrm{p}$ value 0.02 ).

Liver enzymes (AST, ALT and ALP) were significantly elevated in Grade 3 poisoning patients 
much higher than Grade 1 and Grade 2 (p value 0.004, 0.002 and 0.06 respectively) reflecting organ damage due to toxicity of poisoning.
Serum Creatinine levels $(1.04 \pm 0.38) \mathrm{mg} / \mathrm{dl}$ were significantly elevated in grade 3 (p value 0.05 ) than grade 2 and grade 1 (Table 2 )

Other biochemical changes were not significantly altered in different grades of severity.

Table 2: Emergency Laboratory parameters on the day of admission

\begin{tabular}{|l|c|c|c|c|}
\hline \multicolumn{1}{|c|}{ Parameter } & Grade 1 & Grade 2 & Grade 3 & P value \\
\hline Pseudocholinesterase & $7416.25 \pm 1253.99$ & $3496.15 \pm 2020.82$ & $200 \pm 0.00$ & 0.00 \\
\hline WBC & $11.25 \pm 3.62$ & $11.23 \pm 3.16$ & $13.63 \pm 6.06$ & 0.26 \\
\hline RBC & $4.83 \pm 1.11$ & $4.54 \pm 0.77$ & $4.37 \pm 1.01$ & 0.44 \\
\hline Hb & $13.75 \pm 2.49$ & $13.00 \pm 2.64$ & $12.74 \pm 3.28$ & 0.63 \\
\hline Sodium & $140.17 \pm 4.01$ & $139.31 \pm 4.90$ & $140.74 \pm 4.89$ & 0.70 \\
\hline Potassium & $3.92 \pm 0.51$ & $3.85 \pm 0.376$ & $3.47 \pm 0.513$ & 0.026 \\
\hline Chloride & $103.33 \pm 4.6$ & $96.08 \pm 25.81$ & $104.68 \pm 3.87$ & 0.24 \\
\hline Total Bilirubin & $0.92 \pm 0.79$ & $0.46 \pm 0.51$ & $0.68 \pm 0.47$ & 0.16 \\
\hline Total Protein & $7.42 \pm 0.51$ & $7.08 \pm 0.64$ & $7.47 \pm 1.073$ & 3.99 \\
\hline Albumin & $4.33 \pm 0.49$ & $4.15 \pm 0.55$ & $4.05 \pm 0.78$ & 0.50 \\
\hline Globulin & $3.25 \pm 0.62$ & $3.08 \pm 0.27$ & $3.42 \pm 0.50$ & 0.15 \\
\hline AST & $23.25 \pm 7.08$ & $25.85 \pm 6.73$ & $37.95 \pm 17.25$ & 0.004 \\
\hline ALT & $28.42 \pm 7.68$ & $26.62 \pm 8.76$ & $41.68 \pm 16.36$ & 0.002 \\
\hline ALP & $91.33 \pm 21.41$ & $78.46 \pm 31.65$ & $130.68 \pm 92.49$ & 0.068 \\
\hline Urea & $20.92 \pm 5.9$ & $22.31 \pm 8.52$ & $24.47 \pm 11.1$ & 0.566 \\
\hline Creatinine & $0.70 \pm 0.17$ & $0.94 \pm 0.42$ & $1.04 \pm 0.38$ & 0.05 \\
\hline $\begin{array}{l}\text { WBC: White blood cell; RBC: Red blood cell Hb: hemoglobin; AST: Aspartate transaminase; ALT: Alanine } \\
\text { transaminase; ALP: Alkaline phosphatase }\end{array}$ & & & \\
\hline
\end{tabular}

Hyperglycemia was significantly observed in Grade 3 poisoning patients with high RBS levels at the time of admission ( $p$ value 0.03). It was found to be decreased at $6 \mathrm{hrs}$ and $10 \mathrm{hrs}$ of admission but at the time of discharge the RBS values increased. The RBS values were constantly on the higher side in grade 3 at all intervals when compared with Grade 1 and Grade 2 poisoning patients. (Table 3)

Table 3: Comparison of RBS values from time of admission to time of discharge in severity of OP poisoning

\begin{tabular}{|l|c|c|c|c|}
\hline Parameter & Grade 1 & Grade 2 & Grade 3 & P value \\
\hline $\begin{array}{l}\text { RBS (mg/dl) at the time of } \\
\text { admission }\end{array}$ & $103.92 \pm 20.54$ & $112.14 \pm 28.5$ & $139.11 \pm 31.14$ & 0.03 \\
\hline $\begin{array}{l}\text { RBS (mg/dl) } \\
6 \text { hrs after admission }\end{array}$ & $108.15 \pm 15.22$ & $113.43 \pm 16.49$ & $111.72 \pm 22.52$ & 0.93 \\
\hline $\begin{array}{l}\text { RBS(mg/dl) } \\
10 \text { hrs after admission }\end{array}$ & $100.23 \pm 13.29$ & $118.57 \pm 22.14$ & $119.11 \pm 31.79$ & 0.12 \\
\hline $\begin{array}{l}\text { RBS (mg/dl) } \\
\text { Time of discharge }\end{array}$ & $97.85 \pm 14.34$ & $140.14 \pm 34.85$ & $139.17 \pm 21.7$ & 0.01 \\
\hline RBS: Random blood sugar; hrs: hours & & & \\
\hline
\end{tabular}

There was significant Hypokalemia ( $\mathrm{p}$ value 0.02 ) and RBC counts were low (p value 0.02) in patients who have consumed chlorpyriphos OP compound but the pseudocholinesterase levels ( $\mathrm{p}$ value 0.01 ) were lower than in patients who have consumed phorate. (Table 4)

Table 4: Comparison of parameters with different OP compounds

\begin{tabular}{|l|c|c|c|c|c|}
\hline \multicolumn{1}{|c|}{ Parameter } & Chlorpyriphos & Triphos & Cypermethrine & Phorate & p values \\
\hline Potassium & $3.17 \pm 0.408$ & $4.00 \pm 0.00$ & $3.73 \pm 3.75$ & $3.75 \pm 0.44$ & 0.02 \\
\hline Chloride & $86.33 \pm 37.17$ & $103 \pm 4.20$ & $105.27 \pm 3.63$ & $104 \pm 3.56$ & 0.04 \\
\hline Pseudocholinesterase & $2949.33 \pm 337$ & $7331.83 \pm 192$ & $3733.45 \pm 3516$ & $1539 \pm 2168$ & 0.01 \\
\hline RBC & $4.17 \pm 1.32$ & $5.67 \pm 1.21$ & $4.45 \pm 0.82$ & $4.40 \pm 0.68$ & 0.02 \\
\hline RBC: Red blood cell & \multicolumn{5}{|l}{} \\
\hline
\end{tabular}


There was a significant positive correlation between pseudocholinesterase and potassium with $\mathrm{r}$ : 0.334 ( $\mathrm{p}$ value 0.027 ) whereas negative correlation between pseudocholinesterase and AST and ALT with $\mathrm{r}:-0.402$ ( $\mathrm{p}$ value 0.07 ) and $\mathrm{r}:-0.359$ (p: 0.017) respectively. (Graph 1,2,3)

Graph 1: Correlation between pseudocholinesterase and potassium with r: $0.334 \mathrm{p}$ value: 0.027

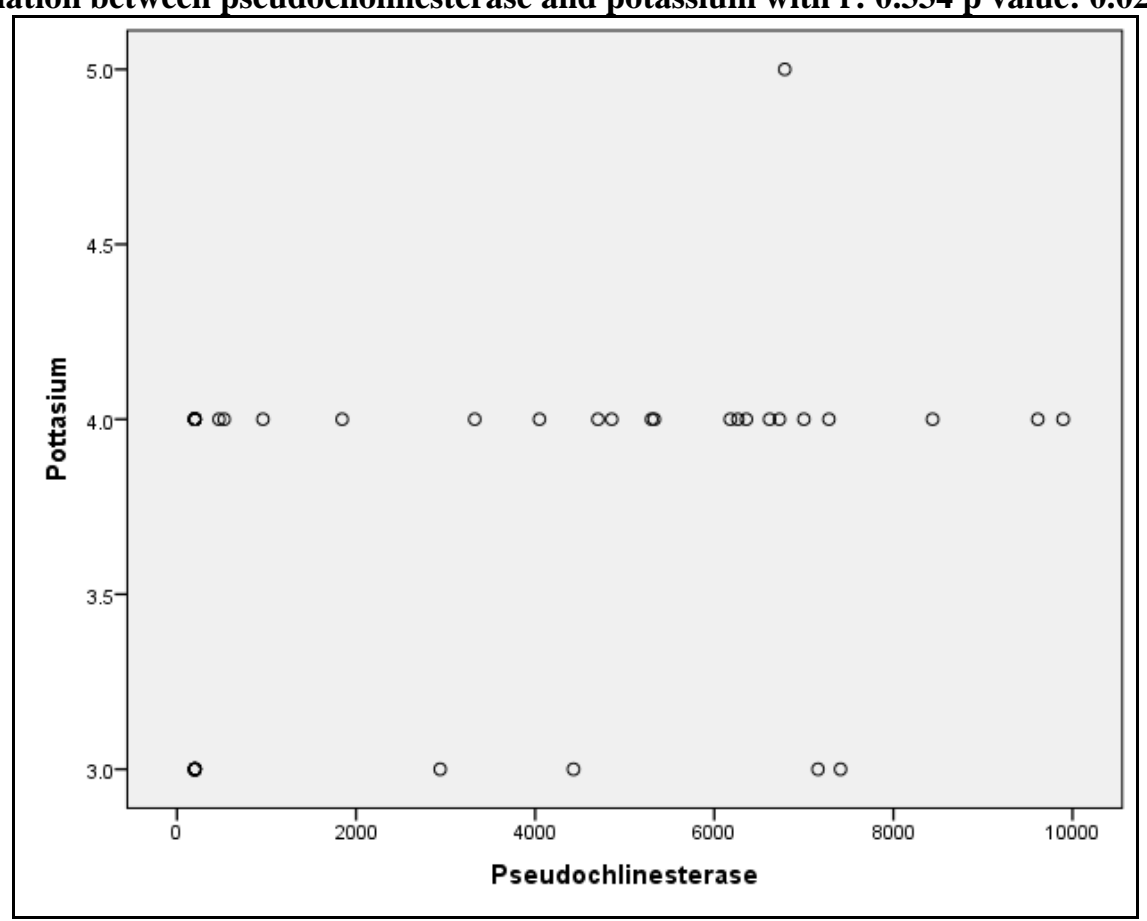

Graph 2: Correlation between pseudocholinesterase and AST with r: -0.402 p value: 0.07

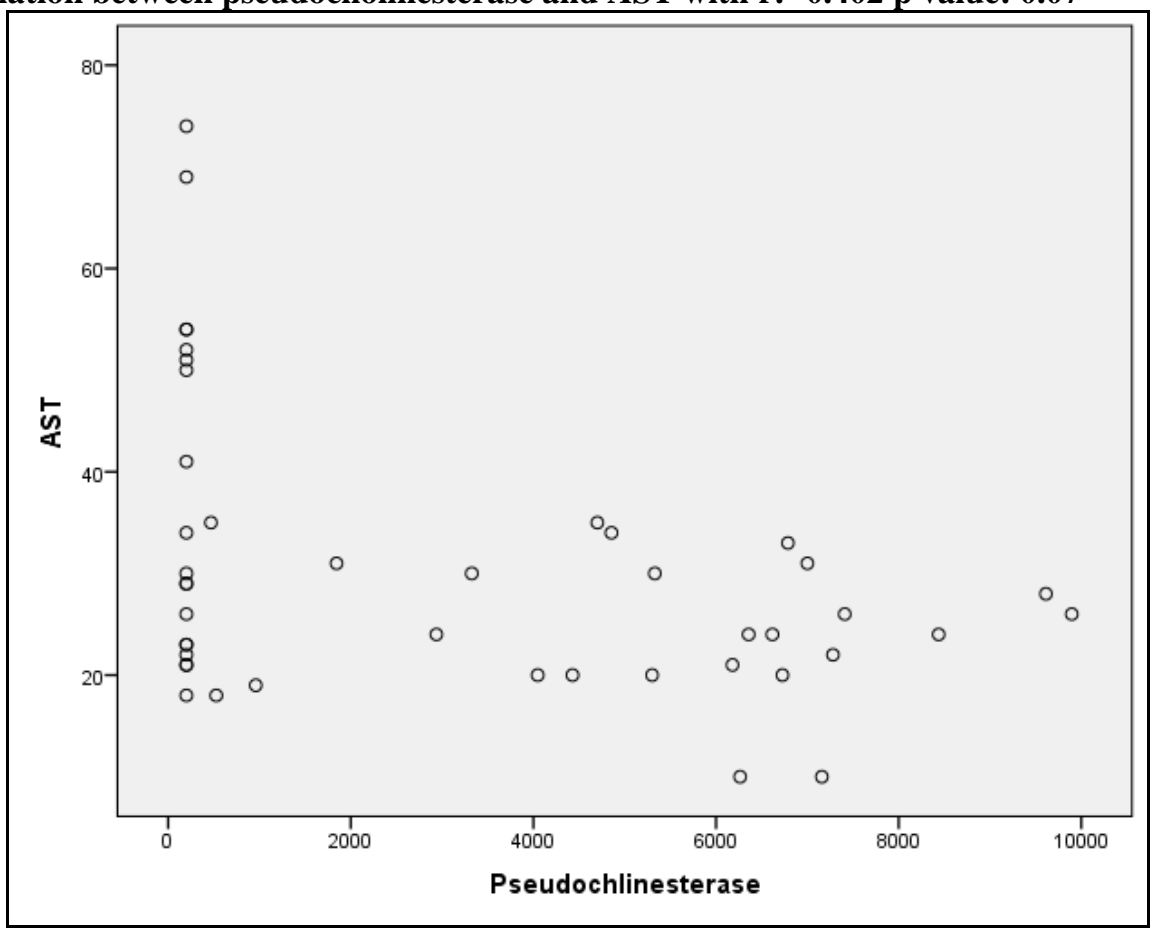


Graph 3: Correlation between pseudocholinesterse and ALT with r: $-\mathbf{0 . 3 5 9} p$ value: 0.017

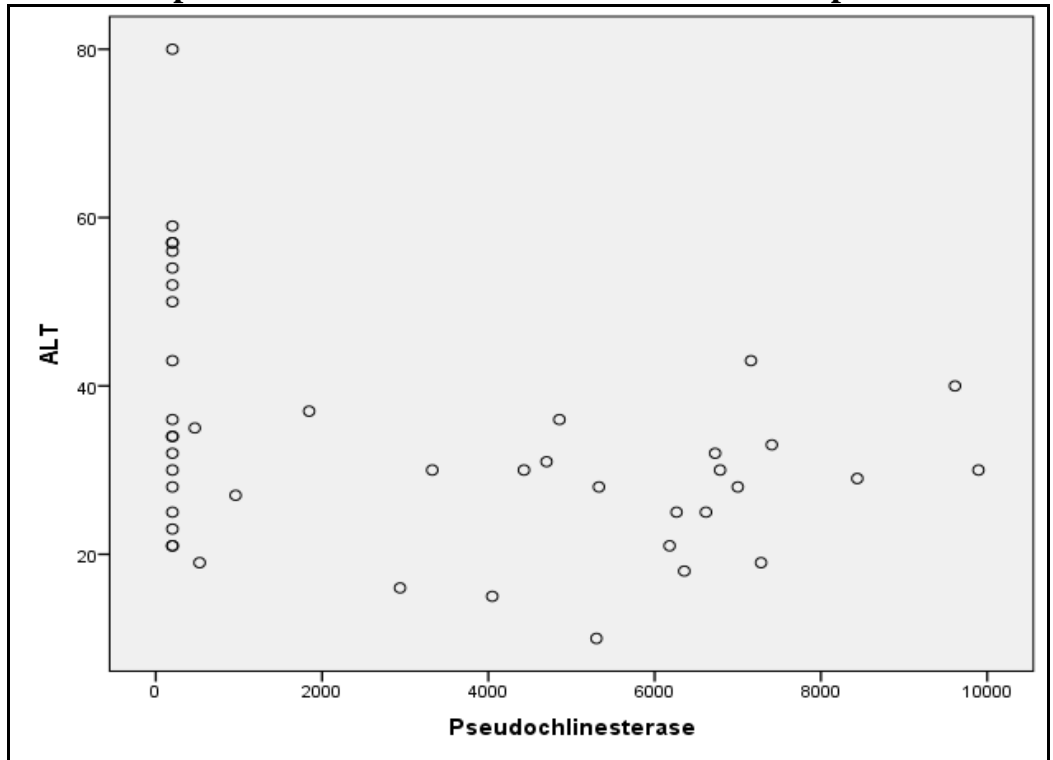

\section{Discussion}

OP poisoning is one of the common medical emergencies in developing countries especially among rural population because of its cheap cost and easy availability. According to WHO one million serious unintentional poisoning occur each year and an additional two million people are hospitalized for suicidal attempts. ${ }^{7}$ In our study all patients consumed the OP poison orally and mode of poisoning was suicidal. (Table 1). As all patients belonged to low socioeconomic status and were from rural background, the common reasons for suicide in these patients were mainly due to financial issues and domestic violence OP compounds apart from inhibiting acetylcholinesterase it also affects the liver, kidney, pancreas and other organfunctions. In our study there was a progressive rise in serum creatinine from Group 1 to Group 3 (Table 2). Various studies ${ }^{1,9}$ have also indicated biochemical derangements due to renal injury the results of Fen You Lee et $\mathrm{al}^{10}$ indicate that patients with OP poisoning have a higher overall incidence and risk of developing acute kidney injury. Elevation in liver enzymes alsooccurs following acute $\mathrm{OP}$ poisoning. ${ }^{1,5,11}$ Experimentally previous studies, ${ }^{1,12,13}$ have demonstrated abnormal liver function tests, hepatic necrosis, and fatty changes in $\mathrm{OP}$ poisoning patients. The biochemical basis for both liver and renal damage is, OP compounds causes oxidative stress and insufficient elimination of free radicals. Free radical injury causes lipid peroxidation (LPO) there by leading to membrane lipid degradation, deterioration of cell integrity and cytotoxic effects. ${ }^{14,15}$

In our study, the RBS levels (Table 3) showed a rising trend from grade1 to grade 3 at the time of admission. Previous studies have concluded that acute pancreatitis may develop following oral ingestion of several organophosphates. Constant elevation of counter regulatory hormones reduces the person's sensitivity to insulin leading to increased blood sugar level. ${ }^{1,3,16}$

There was significant hypokalemia in patients with low pseudocholinesterase level which was similar to Prasad et al study. ${ }^{17}$ Hypokalemia was detected due to renal tubular acidosis ${ }^{18}$ which may aggravate muscle weakness leading to fasciculation and finally respiratory paralysis. (Table 2)

Pseudocholinesterase values were significantly suppressed in Grade 3 patients in comparison to Grade 1 and 2 patients and were similar to results observed in various studies. ${ }^{1,5,19}$ Recent reports ${ }^{20}$ have implied that the degree of plasma cholinesterase inhibition is considerably different in patients consuming different OP compounds. In our study it has been shown that pseudocholinesterase and RBC values were low in patients who have consumed chlorpyriphos and phorate ( $\mathrm{p}$ value 0.01 and 0.02 respectively).

\section{Conclusion}

Emergency laboratory parameters in acute oraganophosphorus poisoning among rural population reflect the need for basic diagnostic facility thereby initiating early treatment to prevent further complications. Laboratory parameters like serum pseudocholinesterase level, renal function tests and liver enzymes concentration can be included in classifying the severity of the poisoning. Inclusion of these biochemical parameters will provide easy and early insight to the conditions of different organs helping accurate clinical judgement and thereby preventing long term complications and morbidity in recovered patients. The knowledge of type of OP compound consumed can predict the severity of OP intoxication and need for emergency intervention 


\section{References}

1. Panda S, Nanda R. Laboratory Abnormalities in Patients with Organophosphorous Poisoning. Indian Medical Gazette. January 2014.

2. Panda S, Nanda R, Mangaraj M, Rathod PK, Mishra PK. Glycemic Status in Oraganophosphorus Poisoning. $J$ Nepal Health Res Counc. 2015;13(31):214-9.

3. Joshi AKR, Rajini PS. Oraganophosphorus insecticides and glucose homeostasis. Food Protectants and Infestation Control Department, Central Food Technological Research Institute (CSIR lab), Mysore, India. February 15th 2012. DOI: 10.5772/28721

4. Fidias E. Leon S, Gustavo Pradilla A, Amboa N, Vesga E. Multiple System Organ Failure, Intermediate Syndrome, Congenital Myasthenic Syndrome, and Anticholinesterase Treatment: The Linkage is Puzzling. Clinical Toxicology. 1996;34(2):245-246.

5. Amanvermez R, Baydin A, Yardan T, Baso N Gunay M. Emergency Laboratory Abnormalities in Suicidal Patients with Acute Organophosphate Poisoning. Turk J Biochem. 2010;35(1):29-34.

6. Bardin PG, Van Eden SF. Organophosphate poisoning: grading the severity and comparing treatment between atropine and glycopyrolate. Crit Care Med. 1990;18:956960.

7. Ravi G, Rajendiran C, Thirumalaikolundusubramanian $\mathrm{P}$, Babu N. Poison control, training and research centre, Institute of Internal Medicine, Government General Hospital, Madras Medical College, Chennai, India. Presented at 6th Annual Congress of Asia Pacific Association of Medical Toxicology, Bangkok, Thailand 2007.

8. Eddleston M and Clark R F. Insecticide: Organophosphates and carbamates in Goldfrank's Toxicologic emergency, $9^{\text {th }}$ ed. Appleton \& Lange; 2006.USA,.1450-1466, 2006.

9. Goswamy R, Chaudhuri A, Mahashur AA. Study of respiratory failure in organophosphate and carbamate poisoning. Heart Lung. 1994;23:466-472.

10. Lee FY, Chen WK, Lin CL. Organophosphate Poisoning and Subsequent Acute Kidney Injury Risk: A Nationwide Population-Based Cohort Study. Malindretos. P, ed. Medicine. 2015;94(47):e2107.

11. Agostini M., Bianchin A. Acute renal failure from organophosphate poisoning: a case of success with haemofiltration. Hum Exp Toxicol, 22:165-167.

12. Yurumez Y, Ikizceli I, Sozuer E.M, Soyuer I, Yavuz Y, Avsarogullarý L, Durukan P. Effect of interleukin-10 on tissue damage caused by organophosphate poisoning. Basic Clin Pharmacol Toxicol. 2007;100:323-327.
13. Kerem M, Bedirli N, Gürbüz N, Ekinci O, BedirliA, Akkaya T, Sakrak O, Pasaoglu H. Effects of acute fenthion toxicity on liver and kidney function and histology in rats. Turk J Med Sci. 2007;37(5):281-288.

14. Rezg R, Mornagui B, El-Fazaa S, Gharbi N. Biochemical evaluation of hepatic damage in subchronic exposure to Malathion in rats: effect on superoxide dismutase and catalase activities using native PAGE. CR Biol. 2008;331(9):655-62.

15. Vandana S. Poovala, Hong Huang, Abdulla K. Salahudeen. Role of Reactive Oxygen Metabolites in Organophosphate-Bidrin-Induced Renal Tubular Cytotoxicity. J Am Soc Nephrol. 1999;10:1746-1752.

16. Clark R.F. Insecticides: Organic phosphorus compounds and carbamates. In: Goldfrank's Toxicologic Emergencies, 7th Edn, The McGray- Hill companies, Inc. 2002 Chapter 88 page: $1346-1357$.

17. Prasad DRMM. Reduced levels of serum potassium and plasma cholinesterase in acute organophosphate poisoning : possible predictive markers. Asia Pac J Med Toxicol. 2014;3:68-72.

18. Narayan R, Abdulla MC, Alungal J. Transient Distal Renal Tubular Acidosis in Organophosphate Poisoning. Indian Journal of Critical Care Medicine: Peer-reviewed, Official Publication of Indian Society of Critical Care Medicine. 2017;21(3):170-171.

19. Kang EJ, Seok S, Lee KH, Gil HW. Factors determining survival in Acute Oraganophosphorus poisoning. KJIM. 2009;24(4):362-366.

20. Eddleston M, Eyer P, Worek F, Sheriff MH, Buckley NA. Predicting outcome using butyrylcholinesterase activity in oraganophosphorus pesticide self-poisoning. $Q J M$. 2008 Jun;101(6):467-74. doi: 10.1093/qjmed/hen026.

How to cite this article: Chandana G,
Rasalkar KP, Reddy GS, Thinakaran V. Study
of emergency laboratory parameters in acute
oraganophosphorus poisoning in a rural
population- retrospective study. Int J Clin
Biochem Res. 2018;5(3):487-492.
Rasalkar KP, Reddy GS, Thinakaran V. Study of emergency laboratory parameters in acute population- retrospective study. Int $\mathrm{J}$ Clin Biochem Res. 2018;5(3):487-492. 